\title{
A Study of Changes in Morphology of Osteoarthritic Articular Cartilage Using Computerized Image Analysis
}

\author{
Neeru Goyal ${ }^{1}$ and Madhur Gupta ${ }^{2}$ \\ ${ }^{1}$ Department of Anatomy, Christian Medical College, Ludhiana, India \\ ${ }^{2}$ Department of Anatomy, Swami Devi Dayal Hospital and Dental College, Golpura, Barwala, \\ Haryana, India
}

Correspondence should be addressed to Neeru Goyal; meetneeru24@yahoo.co.in

Received 5 February 2013; Revised 1 April 2013; Accepted 2 April 2013

Academic Editor: Luigi F. Rodella

Copyright (C) 2013 N. Goyal and M. Gupta. This is an open access article distributed under the Creative Commons Attribution License, which permits unrestricted use, distribution, and reproduction in any medium, provided the original work is properly cited.

\begin{abstract}
Histological studies on articular cartilage have been traditionally based on individual observations but this approach is limited by its subjectivity and bias, yielding considerable variability. So the present study was conducted to observe the various changes in the morphology of osteoarthritic femoral articular cartilage using computerized image analysis. The cartilage specimens were divided into two groups: group $1(n=23)$ (46-81 years) consisted of OA specimens. Group $2(n=12)(41-86$ years) consisted of non-OA specimens. A $5 \mu \mathrm{m}$ thick paraffin sections were stained with H\&E staining and analyzed using Image-Pro Express image analysis software for quantitative analysis of articular cartilage. Various parameters, namely, total thickness of the cartilage, area of lacunae in each zone, area of subchondral cavities, and number of chondrocytes per $10,000 \mu \mathrm{m}^{2}$ area in each zone, were measured. Microscopic appearance of OA cartilage was much different as compared to control. Various changes seen were different in all specimens and they were not related to age. Lacunar size in all four zones was found to differ significantly in the OA (group 1) and control (group 2) $(P<0.05)$. The results suggest that OA should be considered as a specific process and not simply as an inevitable feature of ageing.
\end{abstract}

\section{Introduction}

Articular cartilage undergoes substantial structural and molecular changes with age, including surface fibrillation, alteration of structure and composition of collagen, and decrease in strength. Such changes increase the risk of synovial joint degeneration that leads to osteoarthritis $(\mathrm{OA})$, which is a degenerative joint disease characterized by articular cartilage degeneration. It is the most common of the various articular disorders affecting man. Loss of articular cartilage is the major cause of joint dysfunction and disability in OA.

Since its early development, digital microscopic image analysis has offered the potential for improving the objectivity of microscopic observations. Substantial efforts have already been made to convert the evaluations of experienced pathologists into quantitative values in various research and diagnostic fields [1-3]. In a previous study, using computerized image analysis we observed the changes in the morphology of the non-OA femoral articular cartilage with age and only the lacunar size in zone 3 was found to correlate significantly with age. Despite this difference in lacunar size, normal histology, that is, four zones could be identified in all nonOA specimens though minor changes in the superficial zone, was observed in the ageing articular cartilage [4]. However, in other light microscopic studies $[5,6]$ we observed a completely altered histology throughout the thickness of the cartilage in OA. So the present study was conducted using computerized image analysis to quantitate the differences observed in OA articular cartilage.

\section{Materials and Methods}

Articular cartilage specimens were obtained from the most prominent area of the medial femoral condyle. The specimens were divided into 2 groups. 
TABLE 1: Mean and standard deviation of the total thickness of articular cartilage in OA group.

\begin{tabular}{llllll}
\hline Age group & $41-50$ years & $51-60$ years & $61-70$ years & $71-80$ years & $81-90$ years \\
\hline Total thickness (in $\mathrm{mm})$ & $2.18 \pm 0.02$ & $1.59 \pm 0.54$ & $2.29 \pm 0.55$ & $2.28 \pm 0.14$ & $1.85 \pm 0.24$ \\
\hline
\end{tabular}

TABLE 2: Mean and standard deviation of the area of the lacunae in $\mu \mathrm{m}^{2}$ in OA group.

\begin{tabular}{lcccc}
\hline & Zone 1 & Zone 2 & Zone 3 & Zone 4 \\
\hline 41-50 years & $40.17 \pm 7.63$ & $107.57 \pm 9.42$ & $138.44 \pm 1.98$ & $179.76 \pm 38.42$ \\
51-60 years & $71.66 \pm 25.07$ & $139.63 \pm 22.43$ & $153.88 \pm 61.41$ & $163.02 \pm 59.79$ \\
61-70 years & $78.90 \pm 20.57$ & $142.71 \pm 41.55$ & $161.89 \pm 53.68$ & $227.60 \pm 78.82$ \\
$71-80$ years & $122.12 \pm 47.15$ & $135.94 \pm 44.87$ & $211.04 \pm 40.48$ & $238.96 \pm 115.37$ \\
$81-90$ years & $80.15 \pm 5.23$ & $138.63 \pm 24.22$ & $245.30 \pm 21.32$ & $165.77 \pm 32.22$ \\
\hline
\end{tabular}

2.1. Group $1(n=23)$. This group consisted of OA specimens. The articular cartilage was obtained from patients (46-81 years) of primary OA undergoing total knee replacement in the Department of Orthopaedics, Postgraduate Institute of Medical Education and Research (PGIMER), Chandigarh, India. Patients suffering from rheumatoid arthritis and secondary OA were excluded from the study.

2.2. Group $2(n=12)$. This group (41-86 years) consisted of non-OA specimens. The articular cartilage was obtained from cadavers in the Department of Anatomy within $18 \mathrm{hrs}$ after death. This group served as control group for comparing the primary OA changes as primary OA occurs after 40 years of age. Individuals suffering from any disease of knee joint such as tumor, any deformity, bony enlargement, or irregularities such as osteophytes were excluded from the study.

A $5 \mu \mathrm{m}$ thick serial paraffin sections were stained with haematoxylin and eosin [7] to confirm the histological structure of the articular cartilage and to study the various features for morphometry.

The equipment for morphometric analysis consisted of an Olympus microscope fitted with a digital camera and computer loaded with Image-Pro Express software. In all specimens, every sixth section was observed. At least five sections of each specimen and five fields from each section were observed and photomicrographed. The image acquired on the computer was analyzed using the Image-Pro Express software for the following:

(i) total thickness of the articular cartilage,

(ii) area of lacunae in each zone,

(iii) area of subchondral cavities,

(iv) number of chondrocytes per $10,000 \mu \mathrm{m}^{2}$ area in each zone.

For total thickness of the articular cartilage, sections were photomicrographed at $4 \mathrm{x}$ magnification, the software was calibrated for $4 \mathrm{x}$ magnification, and thickness was measured in millimeters using "measure the distance" option of the software.

For area of lacunae and subchondral cavities, sections were photomicrographed at 40x magnification. The software was calibrated for $40 \mathrm{x}$ magnification, and the area was measured in square microns by drawing the area to be measured using the area of interest (AOI) option of the software. Area of minimum of 1000 cells was measured for every specimen.

For measuring the number of chondrocytes per $10,000 \mu \mathrm{m}^{2}$, photomicrographs at $40 \mathrm{x}$ magnification were used. The software was calibrated for $40 \mathrm{x}$ magnification and the area was measured in square microns by selecting the area. Number of cells in that given area was counted using the "tag points" option of the software.

\section{Statistical Analysis}

SPSS software package was used for statistical analysis. Data was expressed as mean \pm standard deviation. Independent sample $t$-test (Student's $t$-test) was used to determine the statistical significance between the means. Analysis of variance (ANOVA) was done followed by the Student-Newman-Keuls post hoc tests. Partial correlation of various parameters with age was computed using Pearson's correlation test. For all statistical tests, probability levels of less than or equal to $5 \%$ (two-tailed $P$ value $<0.05$ ) were considered to be significant.

\section{Results}

In group 1, that is, the OA cartilage, the articular surface appeared to be very irregular and fibrillated in all the specimens. The cartilage did not show the normal zonation in any of the specimens. Various changes seen were different in all specimens and they were not related to age. The most common feature of the OA articular cartilage was the presence of large clusters of cells (Figure 1). Total thickness of the articular cartilage ranged from 0.52 to $3.17 \mathrm{~mm}$ with an average of $2.02 \pm 0.56 \mathrm{~mm}$ (Table 1 ).

Comparison of the mean of the total thickness at various age groups using ANOVA showed no statistically significant difference in the mean of the total thickness in the OA cartilage specimens. No significant correlation of the total thickness with age was observed.

The area of the lacunae (Table 2) and density of cells (Table 3) were measured. The lacunae were classified as small sized $\left(<100 \mu \mathrm{m}^{2}\right)$, medium sized $\left(100-200 \mu \mathrm{m}^{2}\right)$, and large sized $\left(>200 \mu \mathrm{m}^{2}\right)$ according to area. 
TABLE 3: Mean and standard deviation of the cell density (number of cells per 10,000 $\mu \mathrm{m}^{2}$ area) in all four zones in OA group.

\begin{tabular}{lcccc}
\hline & Zone 1 & Zone 2 & Zone 3 & Zone 4 \\
\hline $41-50$ years & $4.58 \pm 1.05$ & $2.33 \pm 0.45$ & $3.82 \pm 0.24$ & $3.86 \pm 1.36$ \\
$51-60$ years & $5.52 \pm 3.59$ & $3.26 \pm 2.01$ & $3.39 \pm 1.84$ & $3.01 \pm 1.39$ \\
$61-70$ years & $5.53 \pm 2.04$ & $2.49 \pm 0.50$ & $2.55 \pm 0.53$ & $2.33 \pm 0.45$ \\
$71-80$ years & $2.92 \pm 0.95$ & $2.28 \pm 0.76$ & $2.23 \pm 0.33$ & $2.82 \pm 0.43$ \\
$81-90$ years & $1.75 \pm 1.21$ & $1.65 \pm 0.41$ & $2.05 \pm 0.66$ & $1.68 \pm 0.21$ \\
\hline
\end{tabular}

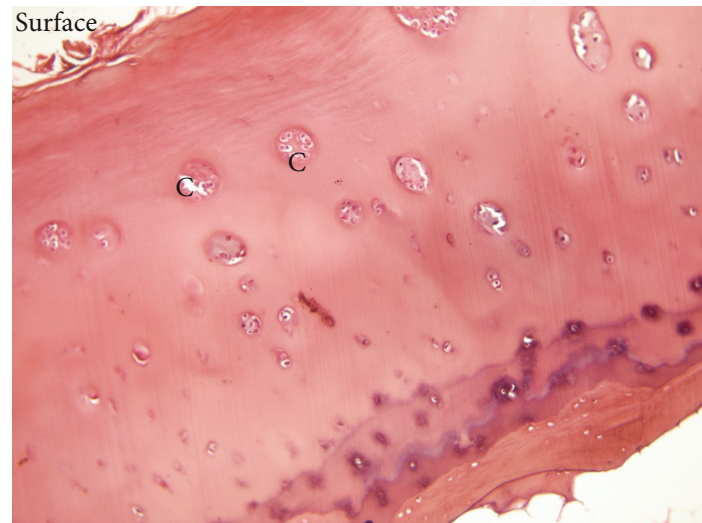

Figure 1: Photomicrograph of osteoarthritic articular cartilage showing irregular fibrillated surface and cell clusters (C). Note that normal zonation into four zones cannot be seen in the osteoarthritic cartilage (H\&E staining, 100x).

4.1. Superficial/Tangential Zone (Zone 1). The area of the lacunae ranged from 34.77 to $165.19 \mu \mathrm{m}^{2}$ with an average of $78.71 \pm 31.12 \mu \mathrm{m}^{2}$. A $77.89 \%$ of the lacunae were small sized $\left(<100 \mu \mathrm{m}^{2}\right)$ in area, $20.60 \%$ were medium sized $\left(100-200 \mu \mathrm{m}^{2}\right)$, and $1.51 \%$ of the lacunae were large sized $\left(>200 \mu \mathrm{m}^{2}\right)$. The density of cells, that is, number of cells per $10,000 \mu \mathrm{m}^{2}$ area, ranged from 1.75 to 13.79 with an average of $4.94 \pm 2.66$.

4.2. Intermediate/Transitional Zone (Zone 2). The area of the lacunae ranged from 88.55 to $224.64 \mu \mathrm{m}^{2}$ with an average of $137.52 \pm 32.70 \mu \mathrm{m}^{2}$. A $28.93 \%$ of the lacunae were small sized, $59.38 \%$ were medium sized, and $11.69 \%$ of the lacunae were large sized. The density of cells ranged from 1.47 to 7.77 with an average of $2.68 \pm 1.29$.

4.3. Radiate/Deep Zone (Zone 3). The area of the lacunae ranged from 77.09 to $254.29 \mu \mathrm{m}^{2}$ with an average of $167.09 \pm$ $55.56 \mu \mathrm{m}^{2}$. A $22.79 \%$ of the lacunae were small sized, $53.12 \%$ were medium sized, and $24.09 \%$ of the lacunae were largesized. The density of cells ranged from 1.43 to 7.77 with an average of $2.89 \pm 1.22$.

4.4. Deepest/Calcified Zone (Zone 4). The area of the lacunae ranged from 63.39 to $384.48 \mu \mathrm{m}^{2}$ with an average of $199.77 \pm$ $76.03 \mu \mathrm{m}^{2}$. A $17.29 \%$ of the lacunae were small sized, $47.89 \%$ were medium sized, and $34.82 \%$ of the lacunae were largesized. The density of cells ranged from 1.24 to 5.66 with an average of $2.73 \pm 1.03$.

4.5. Subchondral Cavities. The area of the subchondral cavities varied from $1,551.02$ to $9,53,428.90 \mu \mathrm{m}^{2}$ with an average of $65,701.07 \pm 204,963.67 \mu \mathrm{m}^{2}$.

Comparison of the mean of various parameters at different age groups using ANOVA showed no statistically significant difference in the various age groups in the OA articular cartilage specimens while the area of lacunae in zone 1 was found to correlate significantly with the age $(P<0.05)$.

In group 2, normal histology was seen, that is, four zones could be identified in all specimens though changes were observed in the superficial zone either in the form of horizontal or oblique splitting of collagen bundles or complete loss of the most superficial zone.

4.6. Comparison of Group 1 and Group 2. On comparing the means using independent sample $t$-test (Student's $t$-test), the means of lacunar size in all four zones were found to differ significantly in the OA (group 1) and control (group 2$)(P<$ 0.05 ). Size of the lacunae in zone 1 and zone 4 was more in OA group while the size of the lacunae in zone 2 and zone 3 was higher in the control group (Table 4).

On applying ANOVA, means of lacunar size in all four zones were found to differ significantly $(P<0.05)$ in group 1 and group 2. On applying post hoc test (Student-NewmanKeuls test), significant difference was found in group 1 (OA group) from the control group.

\section{Discussion}

$\mathrm{OA}$ is often described as a chronic age-related degenerative disease and is considered by many as an inevitable feature of growing old. Rather than OA being a simple consequence of joint ageing and repeated wear and tear, the current concept is that ageing of musculoskeletal system increases the susceptibility to OA but alone does not cause it [8]. The present study also supports the same view point that $\mathrm{OA}$ is not an inevitable feature of ageing. In a previous study [4], using computerized image analysis we compared the morphology of non-OA articular cartilage in below 40 and above 40 years of age and we did not observe much difference in the structure of articular cartilage with age. While a completely altered histology of OA articular cartilage prompted us to compare the parameters from non-OA cartilage above 40 years of 
TABLE 4: Statistical analysis of various parameters in OA and control groups.

\begin{tabular}{|c|c|c|c|c|}
\hline & Group 1 & Group 2 & $T$ value & Significance \\
\hline TT & $2.02 \pm 0.56 \mathrm{~mm}$ & $2.23 \pm 0.36 \mathrm{~mm}$ & 0.099 & NS \\
\hline$A 1$ & $78.71 \pm 31.12$ & $58.92 \pm 18.03$ & 2.023 & Significant \\
\hline$A 2$ & $137.52 \pm 32.70$ & $167.79 \pm 27.18$ & 2.745 & Significant \\
\hline$A 3$ & $167.09 \pm 55.56$ & $204.59 \pm 43.28$ & 2.033 & Significant \\
\hline$A 4$ & $199.77 \pm 76.03$ & $145.00 \pm 32.17$ & 2.374 & Significant \\
\hline$D 1$ & $4.94 \pm 2.66$ & $6.08 \pm 2.97$ & 1.157 & NS \\
\hline$D 2$ & $2.68 \pm 1.29$ & $3.01 \pm 1.35$ & 0.704 & NS \\
\hline D3 & $2.89 \pm 1.22$ & $3.24 \pm 1.11$ & 0.823 & NS \\
\hline$D 4$ & $2.73 \pm 1.03$ & $3.36 \pm 1.37$ & 1.515 & NS \\
\hline ASC & $65,701.07 \pm 204,963.67$ & $4322.89 \pm 2299.21$ & 1.030 & NS \\
\hline
\end{tabular}

$P$ value of less than equal to 0.05 has been considered significant, otherwise nonsignificant (NS). TT: total thickness of the cartilage; $A 1, A 2, A 3$, and $A 4$ : area of lacunae in zone 1-4; D1, D2, D3, and D4: number of chondrocytes per 10,000 $\mu^{2}$ area zone 1-4; ASC: area of subchondral cavities.

age (control group) with $\mathrm{OA}$ articular cartilage as primary osteoarthritis occurs after 40 years of age.

In the present study, structure of the OA articular cartilage was found to be much different from that of control group. The thickness of the OA cartilage was less than that in the control group but this difference was not significant. Articular cartilage thickness has been extensively studied in the knee [9-12]. However, most of these have been performed using radiographic imaging studies, specifically magnetic resonance imaging (MRI). According to Eckstein et al. [10], MRI of strongly curved surfaces can overestimate cartilage thickness without appropriate derivations. Bentley and Hill [13] observed a decrease in cartilage thickness with the increasing severity of OA. They stated that initial changes during OA stimulate a reactive hypertrophy that actually results in an increase in cartilage thickness prior to degradation. After the initial thickening, the articular surface begins to degrade under the influence of degrading enzymes hence causing a final decrease in thickness of the cartilage.

In the present study, the area of lacunae in each zone was measured using image analysis software and it was found to be significantly different in the control and OA group. Significant increase in the size of the lacunae in zone 1 in OA group could actually be a false increase in size. The superficial zone of cartilage is lost during osteoarthritis so these lacunae with increased size could actually belong to zone 2 . Further insights into this observation are required. While an increase in the size of lacunae in zone 4 can be explained as the cells of this zone undergo hypertrophy during $\mathrm{OA}$, decrease in the size of lacunae in zone 2 and 3 in OA could be because the cells form clusters and the newly formed cells could be occupying the smaller lacunae.

No significant difference was observed in the density of chondrocytes in any of the four zones. It could be due to proliferation of chondrocytes in the OA articular cartilage seen in the form of clusters of cells. So the number of cells degenerating due to disease could be almost equal to the newly formed cells forming clusters. This observation signifies that the OA articular cartilage makes an effort to repair but perhaps the regeneration is not at par to compensate for the degenerative changes.
Cake et al. [14] studied the development of OA in ovine knee joints using computer-assisted histomorphometric methods and stated that computerized image analysis showed significant histological differences not detectable by traditional scoring methods. Albano et al. [15] also used computerized image analysis to study the effect of betamethasone on articular cartilage of rabbit knee joints. In the present study, we used the computerized image analysis technique to compare the OA and non-OA articular cartilage in humans.

In the present study, we observed numerous differences in the histological appearance and histomorphometric features of the OA cartilage when compared with ageing cartilage. The various differences observed in the morphology of the $\mathrm{OA}$ and age-matched control articular cartilages suggest that OA should be considered a specific process and not simply an inevitable feature of ageing and the diseased articular cartilage makes an effort to repair itself but these regenerative changes are not at par with the degeneration. A better understanding of the ageing process will help in revealing new therapeutic targets to slow the disease progression.

\section{Conflict of Interests}

The authors declare that they have no conflict of interests.

\section{References}

[1] P. D. Kohlberger, F. Breitenecker, A. Kaider et al., "Modified true-color computer-assisted image analysis versus subjective scoring of estrogen receptor expression in breast cancer: a comparison," Anticancer Research, vol. 19, no. 3, pp. 2189-2193, 1999.

[2] C. F. Chantrain, Y. A. DeClerck, S. Groshen, and G. McNamara, "Computerized quantification of tissue vascularization using high-resolution slide scanning of whole tumor sections," Journal of Histochemistry and Cytochemistry, vol. 51, no. 2, pp. 151-158, 2003.

[3] A. C. Ruifrok, R. L. Katz, and D. A. Johnston, "Comparison of quantification of histochemical staining by hue-saturationintensity (HSI) transformation and color-deconvolution," Applied Immunohistochemistry and Molecular Morphology, vol. 11, no. 1, pp. 85-91, 2003. 
[4] N. Goyal and M. Gupta, "Computerized morphometric analysis of human femoral articular cartilage," ISRN Rheumatology, vol. 2012, Article ID 360201, 6 pages, 2012.

[5] N. Goyal, M. Gupta, K. Joshi, and O. N. Nagi, "Osteoarthritic femoral articular cartilage of knee joint in man," Nepal Medical College Journal, vol. 8, no. 2, pp. 88-92, 2006.

[6] N. Goyal, M. Gupta, K. Joshi, and O. N. Nagi, "Immunohistochemical analysis of ageing and osteoarthritic articular cartilage," Journal of Molecular Histology, vol. 41, no. 4-5, pp. 193-197, 2010.

[7] E. C. Cole, "Studies in Hematoxylin stains," Stain Technology, vol. 18, no. 3, pp. 125-142, 1943.

[8] A. S. Anderson and R. F. Loeser, "Why is osteoarthritis and agerelated disease?" Best Practice \& Research Clinical Rheumatology, vol. 24, no. 1, pp. 15-26, 2010.

[9] C. Adam, F. Eckstein, S. Milz, E. Schulte, C. Becker, and R. Putz, "The distribution of cartilage thickness in the knee-joints of oldaged individuals measurement by A-mode ultrasound," Clinical Biomechanics, vol. 13, no. 1, pp. 1-10, 1998.

[10] F. Eckstein, C. Adam, H. Sittek et al., "Non-invasive determination of cartilage thickness throughout joint surfaces using magnetic resonance imaging," Journal of Biomechanics, vol. 30, no. 3, pp. 285-289, 1997.

[11] B. Kladny, P. Martus, K. H. Schiwy-Bochat, G. Weseloh, and B. Swoboda, "Measurement of cartilage thickness in the human knee-joint by magnetic resonance imaging using a three-dimensional gradient-echo sequence," International Orthopaedics, vol. 23, no. 5, pp. 264-267, 1999.

[12] A. A. Kshirsagar, P. J. Watson, J. A. Tyler, and L. D. Hall, "Measurement of localized cartilage volume and thickness of human knee joints by computer analysis of three-dimensional magnetic resonance images," Investigative Radiology, vol. 33, no. 5, pp. 289-299, 1998.

[13] B. S. Bentley and R. V. Hill, "Assessing macroscopic and microscopic indicators of osteoarthritis in the distal interphalangeal joints: a cadaveric study," Clinical Anatomy, vol. 20, no. 7, pp. 799-807, 2007.

[14] M. A. Cake, R. A. Read, B. Guillou, and P. Ghosh, "Modification of articular cartilage and subchondral bone pathology in an ovine meniscectomy model of osteoarthritis by avocado and soya unsaponifiables (ASU)," Osteoarthritis and Cartilage, vol. 8, no. 6, pp. 404-411, 2009.

[15] M. B. Albano, G. P. Skroch, S. O. Ioshii, X. S. Grahels, P. G. C. de Alencar, and J. E. F. Matias, "Computerized photocolorimetric analysis of the effects of intraarticular betamethasone on the proteoglycan concentration of leporine knee cartilage matrix: influence of the number of intraarticular injections," Revista do Colegio Brasileiro de Cirurgioes, vol. 36, no. 3, pp. 256-260, 2009. 

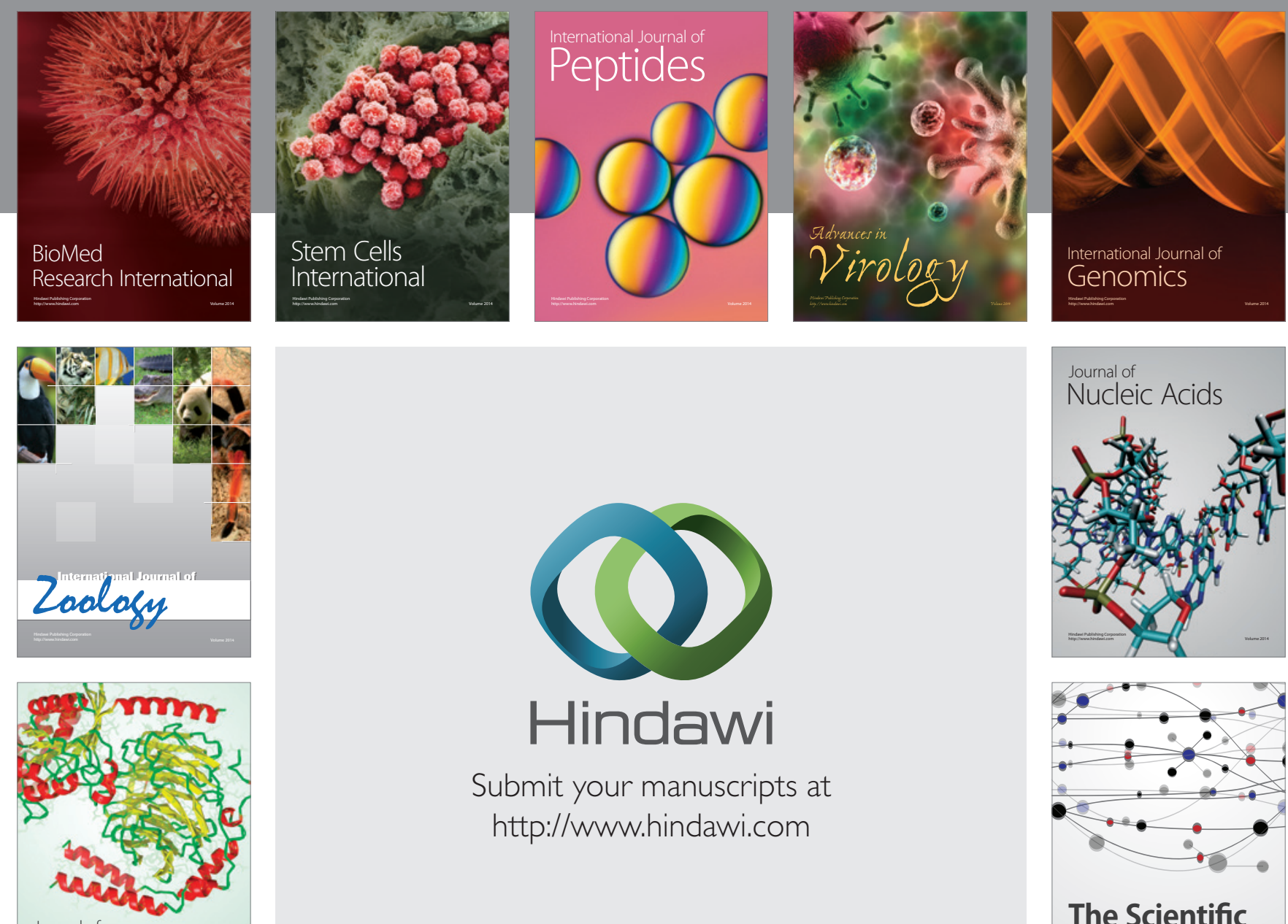

Submit your manuscripts at

http://www.hindawi.com

Journal of
Signal Transduction
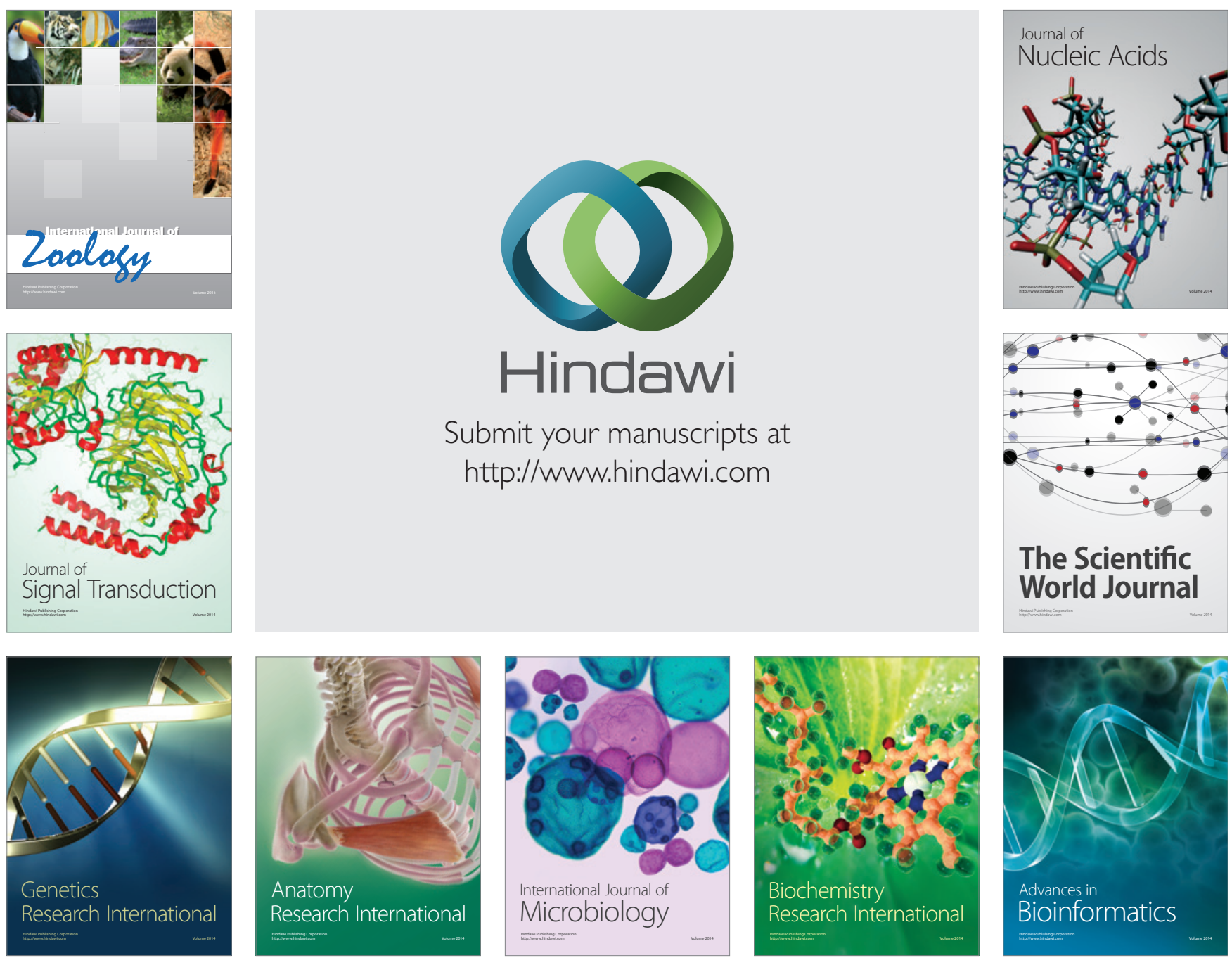

The Scientific World Journal
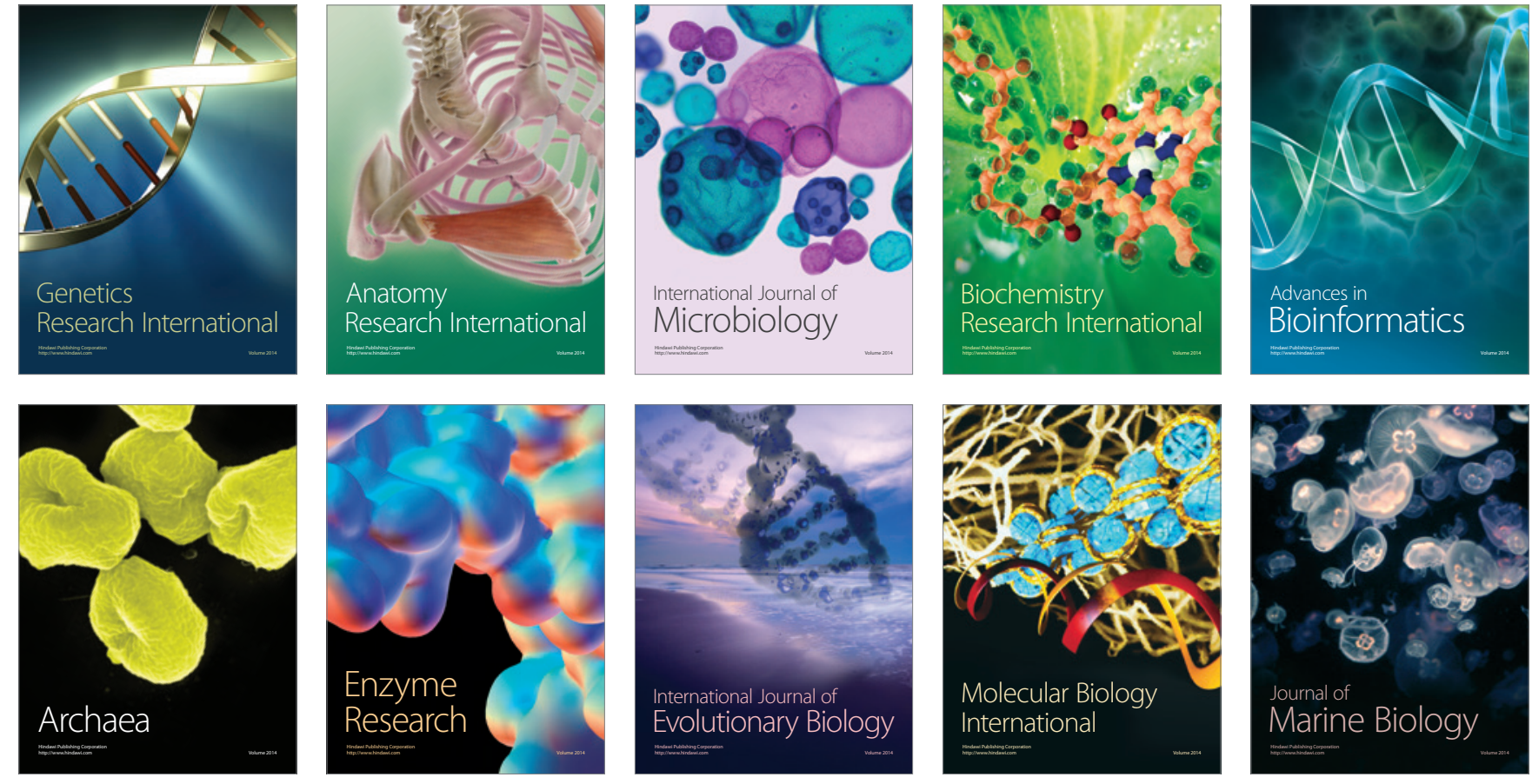